\title{
Impact of environmental changes and land-management practices on wheat production in India
}

\author{
Shilpa Gahlot ${ }^{1}$, Tzu-Shun Lin ${ }^{2}$, Atul K Jain ${ }^{2}$, Somnath Baidya Roy ${ }^{1}$, Vinay K \\ Sehgal $^{3}$, Rajkumar Dhakar ${ }^{3}$ \\ ${ }^{1}$ Centre for Atmospheric Science, Indian Institute of Technology Delhi, New \\ Delhi, 110016 India, \\ ${ }^{2}$ Department of Atmospheric Science, University of Illinois, Urbana, IL, 61801 \\ USA, \\ ${ }^{3}$ Department of Agricultural Physics, Indian Agricultural Research Institute, New \\ Delhi, 110012, India
}

Correspondence to: Somnath Baidya Roy (drsbr@iitd.ac.in)

\section{Online Supplementary Material}

\section{Dynamic C3 crop model in ISAM}

There are six stages in the growth of C3 crop that are modeled in ISAM (Song et al. 2013). The concept of Growing Degree Days (GDD) is used to define wheat growth and divide the wheat-growing season into different phenological stages. Daily GDD is calculated as the difference between daily average air temperature and base temperature since the planting day. Each stage is governed by heat requirement range (fraction of maximum GDD) and switches to the next stage when the heat unit index (=accumulated GDD/maxGDD) exceeds the maximum limit for that stage. GDD is calculated daily and accumulated GDD is calculated as cumulative GDD that increases along the growing season.

During emergence, the seeds stay below the ground, increase in size and gain weight. The emergence of the shoot from the ground marks the beginning of the second stage, the initial vegetative stage. Maximum carbon assimilated is allocated to the shoot in this stage and leads to rapid increase in LAI and a small increase in length of the stem. The third stage, normal vegetative stage, is marked with a rapid increase in length of the stem and the roots to support vertical growth of the plant. The maximum LAI of the crop is reached by the end of the third stage. The fourth stage, initial reproductive stage, marks the onset of reproductive stage in the crop and development of storage organs. Allocation of assimilated carbon to storage organs begins and vegetative development of the plant stops. The fifth stage, post-reproductive stage, marks the solidification of grains and increased nutrient allocation to the grains while ensuring capable roots to support the plant. There are other factors like light availability, temperature stress and nitrogen availability that act as limiting factors to the crop growth and nutrient allocation is promoted in the crop in a way that the impact of these factors is minimized. For instance, there is a greater allocation of carbon to the roots when the crop experiences water stress to ensure more vertical development of roots to extract water from deeper soil levels. Finally, the crop is ready to be harvested when the heat requirement of the crop is fulfilled (GDD= 
maxGDD). Each growth stage is also marked with a maximum number of days that the plant can spend in each stage to ensure that wheat grown in all climatological regions enters each stage based on the defined conditions for each phenology stage.

Total carbon assimilation by vegetation is calculated at hourly intervals using the $\mathrm{C} 3$ photosynthesis (Song et al., 2013) after taking into account the water, nutrient and light availability. Maintenance respiration and growth respiration losses are considered as a part of carbon lost to the environment. Fractions of net carbon assimilated after accounting for respiratory losses are allocated to different plant pools (leaves, stem, coarse root, fine root, and grain) based on the growth stage. 
Table S1: List of all variables/parameters and their values for the spring wheat model. The model equations for C3 crops are described in Song et al. 2013.

\begin{tabular}{|c|c|c|c|}
\hline Symbol & Definition & Value & Source \\
\hline$V_{c m a x 25}$ & $\begin{array}{l}\text { Maximum carboxylation rate at the } \\
\text { reference temperature of } 25^{\circ} \mathrm{C}\end{array}$ & $\begin{array}{l}130 \\
\mu \mathrm{mol} \mathrm{m} \mathrm{m}^{-2} \mathrm{~s}^{-1}\end{array}$ & \\
\hline$T_{\text {high }}$ & $\begin{array}{l}1 / 2 \text { point of high temperature } \\
\text { inhibition function in carbon } \\
\text { assimilation }\end{array}$ & $308 \mathrm{~K}$ & Lokupitiya et al. 2009 \\
\hline$T_{\text {base }}$ & $\begin{array}{l}\text { Base atmospheric temperature for } \\
\text { calculating daily GDD }\end{array}$ & $278.15 \mathrm{~K}$ & Gill et al. 2014 \\
\hline$T_{\text {soil }}$ critical & $\begin{array}{l}\text { Base soil temperature for crop } \\
\text { planting }\end{array}$ & $290.52 \mathrm{~K}$ & \\
\hline$G D D_{\max }$ & $\begin{array}{l}\text { Required total heat above base } \\
\text { temperature }\end{array}$ & $1800\left[{ }^{\circ} \mathrm{C}\right]$ & \\
\hline$H U I_{\text {day }}$ & Heat Unit Index of the $i$ th day & variable & \\
\hline$T_{\text {avg }}$ & Average daily air temperature & variable & \\
\hline$H U I_{v 1}$ & $\begin{array}{l}\text { Minimum heat unit index during } \\
\text { the initial vegetative period }\end{array}$ & 0.07 & This study \\
\hline$H U I_{v 2}$ & $\begin{array}{l}\text { Minimum heat unit index during } \\
\text { the normal vegetative period }\end{array}$ & 0.27 & This study \\
\hline$H U I_{r 1}$ & $\begin{array}{l}\text { Minimum heat unit index during } \\
\text { the reproductive period }\end{array}$ & 0.51 & This study \\
\hline$H U I_{r 2}$ & $\begin{array}{l}\text { Minimum heat unit index during } \\
\text { the post-reproductive period }\end{array}$ & 0.70 & This study \\
\hline$D_{\text {max }_{\text {emer }}}$ & $\begin{array}{l}\text { Maximum number of days in } \\
\text { emergence period }\end{array}$ & 7 & This study (calibrated) \\
\hline$D_{\max _{v 1}}$ & $\begin{array}{l}\text { Maximum number of days in initial } \\
\text { vegetative period }\end{array}$ & 30 & This study(calibrated) \\
\hline$D_{\max _{v 2}}$ & $\begin{array}{l}\text { Maximum number of days in } \\
\text { normal vegetative period }\end{array}$ & 46 & This study(calibrated) \\
\hline$D_{\max _{r 1}}$ & $\begin{array}{l}\text { Maximum number of days in } \\
\text { reproductive period }\end{array}$ & 16 & This study(calibrated) \\
\hline$D_{\max _{r 2}}$ & $\begin{array}{l}\text { Maximum number of days in post- } \\
\text { reproductive period }\end{array}$ & 35 & This study(calibrated) \\
\hline$C_{\text {storage_ref }}$ & $\begin{array}{l}\text { Initial carbon storage in seed as } \\
\text { referenced seeding rate }\end{array}$ & $15 \mathrm{gC}$ & \\
\hline$R_{\text {seed_ref }}$ & Referenced seeding rate & $\begin{array}{l}1011715 \\
\text { seeds/acre }\end{array}$ & \\
\hline$C N_{\text {leaf }}$ & C:N ratio of leaf & 15 & Dreqniak et al. 2013 \\
\hline$C N_{\text {stem }}$ & $\mathrm{C}: \mathrm{N}$ ratio of stem & 50 & Dreqniak et al. 2013 \\
\hline$C N_{\text {root }}$ & $\mathrm{C}: \mathrm{N}$ ratio of root & 30 & Dreqniak et al. 2013 \\
\hline$C N_{\text {grain }}$ & $\mathrm{C}: \mathrm{N}$ ratio of grain & 40 & Dreqniak et al. 2013 \\
\hline Ashoot $_{\boldsymbol{e}}$ & $\begin{array}{l}\text { Allocation fraction for shoot during } \\
\text { emergence period }\end{array}$ & 0.60 & This study(calibrated) \\
\hline Aroot $_{e}$ & $\begin{array}{l}\text { Allocation fraction for root during } \\
\text { emergence period }\end{array}$ & 0.40 & This study(calibrated) \\
\hline$A l_{v 1}$ & $\begin{array}{l}\text { Allocation fraction for leaves } \\
\text { during initial vegetative period }\end{array}$ & 0.45 & This study(calibrated) \\
\hline$A s_{v 1}$ & $\begin{array}{l}\text { Allocation fraction for stem during } \\
\text { initial vegetative period }\end{array}$ & 0.35 & This study(calibrated) \\
\hline$A r_{v 1}$ & Allocation fraction for roots during & 0.20 & This study(calibrated) \\
\hline
\end{tabular}




\begin{tabular}{|c|c|c|c|}
\hline & initial vegetative period & & \\
\hline$A l_{v 2}$ & $\begin{array}{l}\text { Allocation fraction for leaves } \\
\text { during normal vegetative period }\end{array}$ & 0.58 & This study(calibrated) \\
\hline$A s_{v 2}$ & $\begin{array}{l}\text { Allocation fraction for stem during } \\
\text { normal vegetative period }\end{array}$ & 0.32 & This study(calibrated) \\
\hline$A r_{v 2}$ & $\begin{array}{l}\text { Allocation fraction for roots during } \\
\text { normal vegetative period }\end{array}$ & 0.10 & This study(calibrated) \\
\hline$A l_{r 1}$ & $\begin{array}{l}\text { Allocation fraction for leaves } \\
\text { during reproductive period }\end{array}$ & 0.00 & This study(calibrated) \\
\hline$A s_{r 1}$ & $\begin{array}{l}\text { Allocation fraction for stem during } \\
\text { reproductive period }\end{array}$ & 0.05 & This study(calibrated) \\
\hline$A r_{r 1}$ & $\begin{array}{l}\text { Allocation fraction for roots during } \\
\text { reproductive period }\end{array}$ & 0.10 & This study(calibrated) \\
\hline$A g_{r \mathbf{1}_{\max }}$ & $\begin{array}{l}\text { Maximum allocation fraction for } \\
\text { grains during reproductive period }\end{array}$ & 0.85 & This study(calibrated) \\
\hline$A g_{r 1}$ & $\begin{array}{l}\text { Allocation fraction for grains } \\
\text { during reproductive period }\end{array}$ & variable & This study \\
\hline$A l_{r 2}$ & $\begin{array}{l}\text { Allocation fraction for leaves } \\
\text { during post-reproductive period }\end{array}$ & 0.00 & This study(calibrated) \\
\hline$A s_{r 2}$ & $\begin{array}{l}\text { Allocation fraction for stem during } \\
\text { post-reproductive period }\end{array}$ & 0.00 & This study(calibrated) \\
\hline$A r_{r 2}$ & $\begin{array}{l}\text { Allocation fraction for roots during } \\
\text { post-reproductive period }\end{array}$ & 0.05 & This study(calibrated) \\
\hline$A g_{r 2_{\text {max }}}$ & $\begin{array}{l}\text { Allocation fraction for grains } \\
\text { during post-reproductive period }\end{array}$ & 0.95 & This study(calibrated) \\
\hline$A g_{r 2}$ & $\begin{array}{l}\text { Allocation fraction for grains } \\
\text { during post-reproductive period }\end{array}$ & variable & This study \\
\hline$T_{\min }^{\text {stress }}$ & $\begin{array}{l}\text { Minimum temperature for inducing } \\
\text { heat stress in the crop }\end{array}$ & $25^{\circ} \mathrm{C}$ & Deryng et al. 2014 \\
\hline$T_{\max }^{\text {stress }}$ & $\begin{array}{l}\text { Maximum temperature for wheat to } \\
\text { have non-zero daily grain growth } \\
\text { rate }\end{array}$ & $35^{\circ} \mathrm{C}$ & Deryng et al. 2014 \\
\hline$R t_{\text {high }}$ & $\begin{array}{l}\text { Daily death rate of leaves from heat } \\
\text { stress }\end{array}$ & variable & This study \\
\hline SLA & Specific Leaf Area & $45 \mathrm{~m}^{2} / \mathrm{kgC}$ & This study \\
\hline$H_{a}$ & Maximum canopy height & $0.95 \mathrm{~m}$ & Dreqniak et al. 2013 \\
\hline phen & Phenology Stage & variable & \\
\hline$T_{\text {stress }}$ fact & $\begin{array}{l}\text { Daily heat stress factor due to high } \\
T_{\text {avg }}\end{array}$ & variable & \\
\hline day & Julian day & variable & \\
\hline$R t_{\max }$ & $\begin{array}{l}\text { Maximum death rate of green } \\
\text { leaves due to heat stress }\end{array}$ & variable & \\
\hline$L A I_{\max }$ & Maximum LAI & 7.0 & Dreqniak et al. 2013 \\
\hline
\end{tabular}


Table S2: Climatology based planting day criteria for each grid cell. (*Average minimum temperature based on 1901-1950 climatology).

\begin{tabular}{|c|c|c|c|}
\hline $\begin{array}{l}\text { Minimum } \\
\text { temperature* } \\
\text { of region } \\
\left(T_{\text {air }} \text { min }\right. \\
\end{array}$ & Criteria & Notation & $\begin{array}{l}\text { Spatial regions } \\
\text { represented }\end{array}$ \\
\hline$T_{a i r_{\min }}<275.0$ & $\begin{array}{l}\text { - Julian day }>260 \\
\text { (September } 17 \text { ) } \\
\text { - Average of last } 7 \text { day air } \\
\text { temperature }<24.81^{\circ} \mathrm{C}\end{array}$ & $\begin{array}{l}\text { day }>260 \\
T_{\text {air }}(7 \text { days })<297.96 \mathrm{~K}\end{array}$ & $\begin{array}{l}\text { - Northern parts of } \\
\text { India } \\
\text { - Indo-Gangetic } \\
\text { Plains }\end{array}$ \\
\hline $\begin{array}{l}275.0 \leq T_{\text {air }} \text { min } \\
<277.5\end{array}$ & $\begin{array}{l}\text { - Julian day }>260 \\
\text { (September } 17 \text { ) } \\
\text { - Average of last } 7 \text { day air } \\
\text { temperature }<22.81^{\circ} \mathrm{C} \\
\text { - Average of last } 30 \text { days } \\
\text { precipitation }<20 \mathrm{~mm}\end{array}$ & $\begin{array}{l}\text { day }>260 \\
T_{\text {air }}(7 \text { days })<295.96 \mathrm{~K} \\
\operatorname{Prec}(30 \text { days })<20 \mathrm{~mm}\end{array}$ & $\begin{array}{l}\text { - Parts of eastern } \\
\text { India where rice is } \\
\text { harvested a few } \\
\text { months ahead of } \\
\text { wheat plantation on } \\
\text { the same land. } \\
\end{array}$ \\
\hline 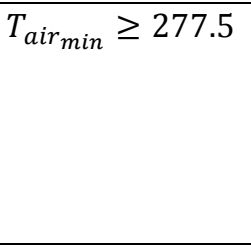 & $\begin{array}{l}\text { - Julian day }>260 \\
\text { (September } 17 \text { ) } \\
\text { - Average of last } 7 \text { day air } \\
\text { temperature }<32.01^{\circ} \mathrm{C} \\
\text { - Average of last } 30 \text { days } \\
\text { precipitation }<100 \mathrm{~mm}\end{array}$ & $\begin{array}{l}\text { day }>260 \\
T_{\text {air }}(7 \text { days })<305.16 \mathrm{~K} \\
\\
\operatorname{Prec}(30 \text { days }) \\
<100 \mathrm{~mm}\end{array}$ & $\begin{array}{l}\text { - Central India and } \\
\text { parts of southern } \\
\text { India. }\end{array}$ \\
\hline
\end{tabular}


Table S3: List of equations for spring wheat specific processes and variables used in this study. The variables used here are defined in Table S4.

\begin{tabular}{|c|c|}
\hline $\begin{array}{l}\text { Dataset/Proces } \\
\text { s/Variable }\end{array}$ & Equation \\
\hline Heat Stress & 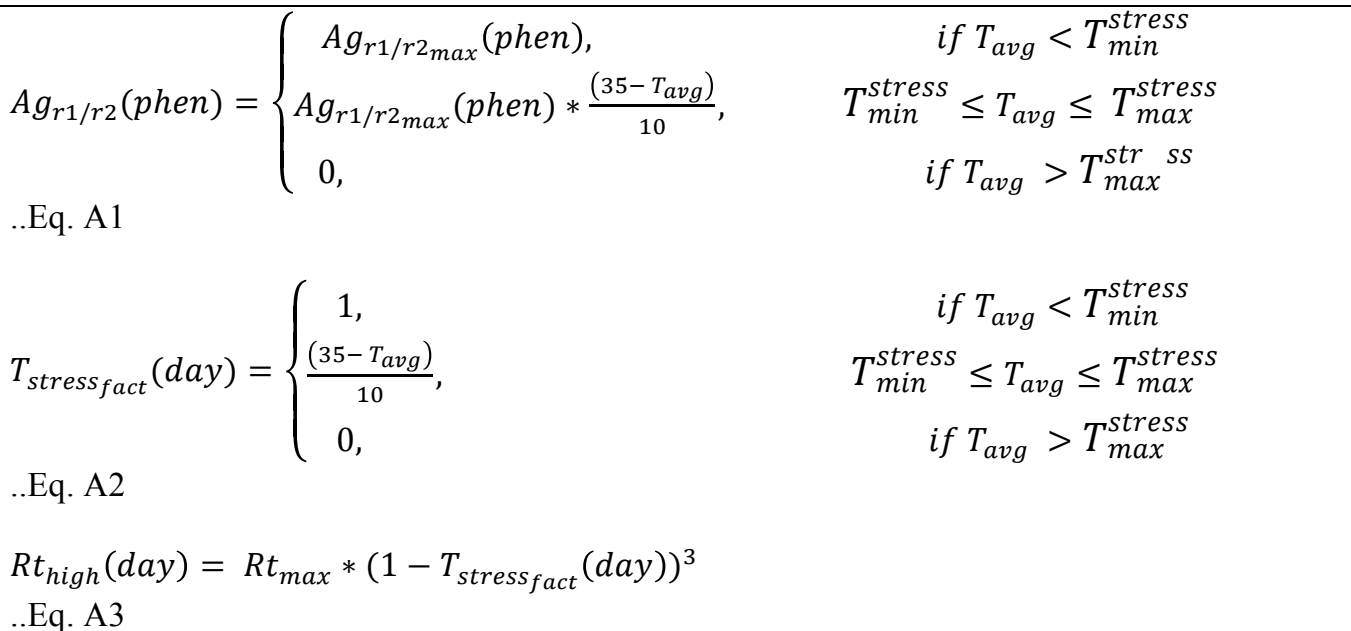 \\
\hline $\begin{array}{l}\text { Annual nitrogen } \\
\text { fertilizer data } \\
\left(0.5^{\circ} \times 0.5^{\circ}\right) \text { from } \\
1900 \text { to } 2005\end{array}$ & $\begin{array}{l}\text { frac }_{i}=\frac{\text { fert }_{\text {wheat }_{i}}(2000)}{\text { fert }_{\text {total }_{i}}(2000)} \\
\text {..Eq. A4 } \\
\text { fert }_{\text {wheat }_{i}}(y r)=\text { frac }_{i} * \text { fert }_{\text {total }_{i}}(y r) \\
\text {..Eq. A5 }\end{array}$ \\
\hline $\begin{array}{l}\text { Annual wheat area } \\
\text { data } \\
\left(0.5^{\circ} \times 0.5^{\circ}\right) \\
\text { from } 1997 \text { to } 2014\end{array}$ & $\begin{array}{l}\text { Area }_{w_{\text {heat }}} \text { state }(y r)=\sum_{k=1}^{\text {all districts }} \text { Area }_{\text {wheat }_{k}}(y r) \\
\text {..Eq. A6 } \\
\text { fr }_{\text {state }_{y r}}=\frac{\text { Area }_{\text {wheat }_{\text {state }}(y r)}}{\text { Area }_{\text {heat }} \text { state }^{(2000)}} \\
\text {..Eq. A7 } \\
T W A_{i}(y r)=\text { fr }_{\text {state }_{y r}} * T W A_{i}(2000), \forall i \in \text { state } \\
\text {..Eq. A8 }\end{array}$ \\
\hline $\begin{array}{l}\text { Annual area } \\
\text { equipped for } \\
\text { irrigation } \\
\left(0.5^{\circ} \times 0.5^{\circ}\right) \\
\text { from } 1997 \text { to } 2014\end{array}$ & 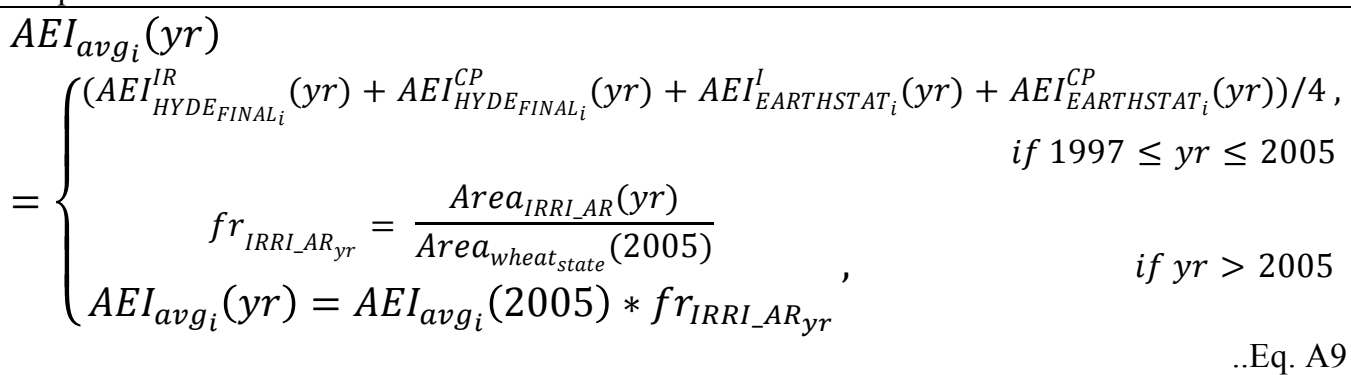 \\
\hline $\begin{array}{l}\text { Annual actual } \\
\text { wheat production }\end{array}$ & $\begin{array}{l}\operatorname{Prod}_{\text {act }}(y r)=\frac{\sum_{i=1}^{\text {all grids }}\left\{\left[\operatorname{Prod}_{S_{C O N}(y r)_{i} * A E I} v_{g}(y r)_{i}\right]+\left[\operatorname{Prod}_{S_{I R I I}}(y r)_{i} *\left[T W A(y r)_{i}-A E I_{a v g}(y r)_{i}\right]\right]\right\}}{\sum_{i=1}^{\text {all grids }} T W A(y r)_{i}} \\
\text {..Eq. A10 }\end{array}$ \\
\hline $\begin{array}{l}\text { Impact of each } \\
\text { factor on wheat } \\
\text { production }\end{array}$ & $\begin{array}{l}\operatorname{Impact} \\
\text {..Eq. A11 } 1 \text {. }\end{array}$ \\
\hline
\end{tabular}


Table S4: Definition of all variables and parameters used in the equations presented in Table S3.

\begin{tabular}{|c|c|c|}
\hline Symbol & Definition & Source \\
\hline$y r$ & Year & \\
\hline$i$ & $\begin{array}{l}\text { Variable representing number of grids in study } \\
\text { area }\end{array}$ & \\
\hline$k$ & $\begin{array}{l}\text { Variable representing number of districts in a } \\
\text { state }\end{array}$ & \\
\hline Prod $_{\text {act }}$ & Actual annual wheat production of India & \\
\hline$S_{\text {CON }}$ & $\begin{array}{l}\text { Control run with all input forcings (atmospheric } \\
\mathrm{CO}_{2} \text {, temperature, nitrogen fertilizer, irrigation) } \\
\text { varying with time }\end{array}$ & \\
\hline$S_{<\text {factor }>}$ & $\begin{array}{l}\text { Simulations with all but one input forcing } \\
\text { (factor) varying with time }\end{array}$ & \\
\hline $\operatorname{Prod}_{S_{C O N}}$ & $\begin{array}{l}\text { Wheat production from } S_{C O N} \text { case (irrigated } \\
\text { case) }\end{array}$ & \\
\hline Prod $_{S_{<\text {factor }>}}$ & Wheat production from $S_{<\text {factor }>}$ case & \\
\hline Area $_{\text {wheat }_{k}}(y r)$ & Annual wheat harvested area at district $(k)$ level & MAFW, India \\
\hline Area $_{\text {wheat }}$ state & $\begin{array}{l}\text { Annual wheat harvested area at state level } \\
\text { evaluated by summing up data from all districts } \\
\text { in a state }\end{array}$ & \\
\hline fr $_{\text {state }_{y r}}$ & $\begin{array}{l}\text { Fraction of annual wheat harvested area at } \\
\text { state-level for year } y r \text { with that of year } 2000 \text {. }\end{array}$ & \\
\hline$T W A_{i}$ & Total wheat harvested area in $i$ th grid & This study \\
\hline$A E I_{H Y D E_{F I N A L_{i}}^{I R}}$ & $\begin{array}{l}\text { Gridded Area Equipped for Irrigation (AEI) } \\
\text { with HYDE } 3.1 \text { Final as dataset used for } \\
\text { downscaling and maximizing consistency with } \\
\text { AEI_IR }\end{array}$ & Siebert et al. 2015 \\
\hline$A E I_{H Y D E_{F I N A L i}}^{C P}$ & $\begin{array}{l}\text { Gridded Area Equipped for Irrigation (AEI) } \\
\text { with HYDE } 3.1 \text { Final as dataset used for } \\
\text { downscaling and maximizing consistency with } \\
\text { AEI CP }\end{array}$ & Siebert et al. 2015 \\
\hline$A E I_{\text {EARTHSTA }}^{I R}{ }_{i}$ & $\begin{array}{l}\text { Gridded Area Equipped for Irrigation (AEI) } \\
\text { with EARTHSTAT as dataset used for } \\
\text { downscaling and maximizing consistency with } \\
\text { AEI IR }\end{array}$ & Siebert et al. 2015 \\
\hline$A E I_{E A R T H S T A T_{i}}^{C P}$ & $\begin{array}{l}\text { Gridded Area Equipped for Irrigation (AEI) } \\
\text { with EARTHSTAT as dataset used for } \\
\text { downscaling and maximizing consistency with } \\
\text { AEI_CP }\end{array}$ & Siebert et al. 2015 \\
\hline$A E I_{\text {avg }}$ & Average AEI for each grid cell & \\
\hline $\operatorname{frac}_{i}$ & $\begin{array}{l}\text { Fraction of wheat to total fertilizer amount for } \\
i \text { th grid }\end{array}$ & \\
\hline fert $_{\text {wheat }_{i}}(y r)$ & $\begin{array}{l}\text { Fertilizer amount added to wheat for } i \text { th grid } \\
\text { for the year } y r\end{array}$ & \\
\hline fert $_{\text {total }_{i}}(y r)$ & $\begin{array}{l}\text { Total fertilizer amount added in } i \text { th grid for the } \\
\text { year } y r\end{array}$ & \\
\hline Impact $_{\text {factor }}$ & $\begin{array}{l}\text { Difference between production from } S_{C O N} \text { and } \\
S_{<\text {factor }>}\end{array}$ & \\
\hline
\end{tabular}

